\title{
Assessment of adult formulas for glomerular filtration rate estimation in children
}

\author{
Hassib Chehade • Eric Girardin • Katia Iglesias • \\ Pascal Ramseyer • Peter Frey • Daniel Bardy • \\ Dolores Mosig $•$ Francois Cachat
}

Received: 11 March 2012 /Revised: 18 July 2012 / Accepted: 6 August 2012 /Published online: 13 September 2012

(C) IPNA 2012

\begin{abstract}
Background Estimated glomerular filtration rate (eGFR) is an important diagnostic instrument in clinical practice. The National Kidney Foundation-Kidney Disease Quality Initiative (NKF-KDOQI) guidelines do not recommend using formulas developed for adults to estimate GFR in children; however, studies confirming these recommendations are scarce. The aim of our study was to evaluate the accuracy of the new Chronic Kidney Disease Epidemiology Collaboration (CKD-EPI) formula, the Modification of Diet in Renal Disease (MDRD) formula, and the Cockcroft-Gault formula in children with various stages of chronic kidney disease (CKD).

Methods A total of 550 inulin clearance (iGFR) measurements for 391 children were analyzed. The cohort was divided
\end{abstract}

into three groups: group 1, with iGFR $>90 \mathrm{ml} / \mathrm{min} / 1.73 \mathrm{~m}^{2}$; group 2, with iGFR between 60 and $90 \mathrm{ml} / \mathrm{min} / 1.73 \mathrm{~m}^{2}$; group 3 , with iGFR of $<60 \mathrm{ml} / \mathrm{min} / 1.73 \mathrm{~m}^{2}$.

Results All formulas overestimate iGFR with a significant bias $(p<0.001)$, present poor accuracies, and have poor Spearman correlations. For an accuracy of $10 \%$, only 11 , 6 , and $27 \%$ of the eGFRs are accurate when using the MDRD, CKD-EPI, and Cockcroft-Gault formulas, respectively. For an accuracy of $30 \%$, these formulas do not reach the NKF-KDOQI guidelines for validation, with only 25 , 20 , and $70 \%$ of the eGFRs, respectively, being accurate.

Conclusions Based on our results, the performances of all of these formulas are unreliable for eGFR in children across all CKD stages and cannot therefore be applied in the pediatric population group.

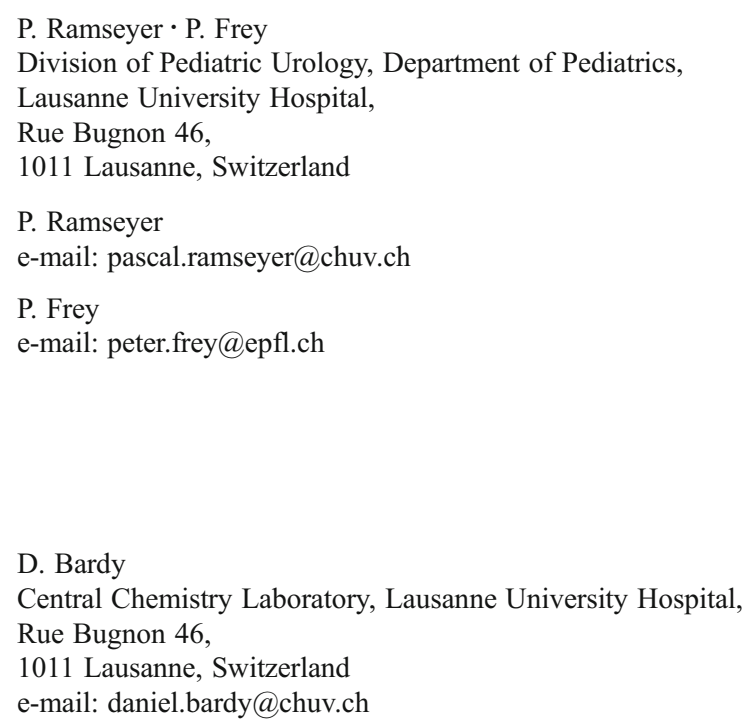


Keywords Glomerular filtration rate - MDRD formula . CKD-EPI formula $\cdot$ Cockcroft-Gault formula $\cdot$ Children . Chronic kidney disease

\section{Introduction}

An exact evaluation of the glomerular filtration rate (GFR) is clinically important in adults and children when kidney function is being evaluated, but it can be difficult and cumbersome to realize $[1,2]$. The method for assessing exact GFR requires the measurement of an exogenous marker, such as inulin, ${ }^{125}$ iothalamate, iohexol, or chrome-EDTA. However, these methods are relatively invasive and time-consuming, and they may impose significant constraints, especially on children. Therefore, several bedside formulas using endogenous markers, such as serum creatinine $(\mathrm{Cr})$ have been developed to obtain an estimated GFR (eGFR). Each of these formulas was initially developed in a selective population, such as children with mild to moderate renal failure or adults with chronic kidney diseases (CKD). The most commonly used Cr-based GFR formulas in adults are the CockcroftGault formula [3], the Modification of Diet in Renal Disease (MDRD) formula [4] and, more recently, the Chronic Kidney Disease Epidemiology Collaboration (CKD-EPI) formula [5]. These formulas have proved to be reliable for obtaining the eGFR in a large population of adults, but studies for their application and reliability in children are scarce and have been only performed using the Cockcroft-Gault and the MDRD formulas, with conflicting results. Pierrat et al. [6] found that the Cockcroft-Gault formula is appropriate for estimating GFR in children older than 12 years and that the MDRD formula presents a large overestimation in all children above the age of 3 years. However, Filler et al. [7] concluded that the Cockcroft-Gault formula provides a poor estimate of the GFR in children aged 1 to 18 years, with a considerable bias, and that it should therefore not be used in this population group. The aim of our study was to evaluate the accuracy of the two recently published adult formulas (MDRD and CKD-EPI), and well as the Cockcroft-Gault formula, in children with various stages of CKD by comparing them to the gold standard method of GFR measurement, which is inulin clearance (iGFR).

\section{Materials and methods}

Population

This study was approved by the local research ethics board under the number 300/11. We retrospectively analyzed 550 measurements of inulin clearance for 391 children (age range 3-18 years; all white except for 2 blacks) determined between 2005 and 2010. Our study population was divided into three groups: group 1, with iGFRs $>90 \mathrm{ml} / \mathrm{min} / 1.73 \mathrm{~m}^{2}$ (CKD stage I); group 2, with iGFRs between 60 and $90 \mathrm{ml} / \mathrm{min} / 1.73 \mathrm{~m}^{2}$ (CKD stage II); group 3, with iGFRs $<60 \mathrm{ml} / \mathrm{min} / 1.73 \mathrm{~m}^{2}$ (CKD stage III, IV, and V). Demographic characteristics of the patients are summarized in Table 1 for the whole sample and by stages of CKD. Inulin clearance was measured in children with various kidney disorders, including obstructive or reflux uropathy, congenital or acquired single kidney, glomerulonephritis, nephrotic syndrome, polycystic kidney disease (autosomal dominant or recessive), hemolytic uremic syndrome, and other diseases, as reported in Table 2. Indications for clearance performance were left at the discretion of the referring physician (pediatric nephrologists or urologists). Excluded from the study were children with bladder dysfunction, those unable to void spontaneously, and those in whom bladder catheterization failed. Proper emptying of the bladder was evaluated by comparing the urine output with its osmolality. A decreasing diuresis with a concomitant decreasing urine osmolality was an indication of poor bladder emptying, and the child was then excluded from the study for technical reasons. All patients fasted the night before the day of the investigation, and drugs interfering with the inulin measurement were not used before and during the test. Height ( $\mathrm{Ht}$; to the nearest centimeter) and body weight (BW; to the nearest gram) were recorded, and a baseline blood sample was drawn to determine the serum $\mathrm{Cr}$ level.

Table 1 Demographic characteristics

\begin{tabular}{lrcrrrr}
\hline CKD stages & $n$ & Number of females (\%) & Median age (years) & Age range (years) & Median Ht Z-score & Median BW Z-score \\
\hline CKD stage I & 303 & $130(43)$ & $11.3[8.0 ; 14.4]$ & $3-18.0$ & $0.19[-0.55 ; 1.07]$ & $0.11[-0.63 ; 0.91]$ \\
CKD stage II & 180 & $75(42)$ & $12.5[9.4 ; 15.4]$ & $3.5-18.0$ & $-0.04[-0.87 ; 0.84]$ & $-0.23[-0.92 ; 0.69]$ \\
CKD stage III, IV and V & 67 & $39(58)$ & $13.1[9.4 ; 16.1]$ & $4.2-18.0$ & $-0.97[-2.04 ; 0.25]$ & $-1.13[-2.20 ;-0.15]$ \\
Total & 550 & $244(44)$ & $11.7[8.4 ; 14.9]$ & $2.9-18.0$ & $0.00[-0.85 ; 0.85]$ & $-0.13[-0.87 ; 0.78]$ \\
\hline
\end{tabular}

BW, Body weight; CKD, chronic kidney disease; Ht, height

Unless otherwise indicated, data are presented as the median, with the upper and lower quartiles (25\%; $75 \%)$ given in square parenthesis 
Table 2 Disease category

\begin{tabular}{|c|c|c|c|c|}
\hline & Etiologies & CKD stage I $(n)$ & CKD stage II $(n)$ & $\begin{array}{l}\text { CKD stages III, } \\
\text { IV and V }(n)\end{array}$ \\
\hline & Obstructive or reflux uropathy & 150 & 86 & 13 \\
\hline & Congenital and acquired single kidney & 57 & 38 & 4 \\
\hline & Polycystic kidney disease & 9 & 6 & 4 \\
\hline & Glomerulonephritis & 19 & 8 & 6 \\
\hline & Hemolytic and uremic syndrome & 1 & 8 & 5 \\
\hline & Metabolic disease & 2 & 4 & 8 \\
\hline & Post-chemotherapy & 6 & 6 & 11 \\
\hline & Pyelonephritis/recurrent urinary tract infections & 12 & 6 & 0 \\
\hline & Other & 48 & 18 & 16 \\
\hline & Total: 550 & 303 & 180 & 67 \\
\hline
\end{tabular}

CKD, chronic kidney disease

\section{Laboratory methods}

\section{Serum Cr calibration}

Serum creatinine (SCr) level was measured using the kinetic colorimetric compensated Jaffe method as reported by the manufacturer (Roche Modular P system; Roche Diagnostics, Mannheim, Germany) and was closely aligned with the values determined by enzymatic assay and standardized against the reference isotope dilution mass spectrometry method (IDMS). We compared the compensated Jaffe technique and the enzymatic method in tests conducted in our laboratory to verify this correlation (Fig. 1). Our results showed an average difference of 0.99 and a $95 \%$ confidence interval (CI) of -6.586 to 8.566 . The method was calibrated with the calibrator using procedures described by Roche Diagnostics. The IDMS traceability involves a two-point calibration (target values at 0 and 360-390 $\mu \mathrm{mol} / 1$, depending on the calibrator lot) and the subtraction of $26 \mu \mathrm{mol} / 1$ from the results as compensation for the non-specific chromogens. The inter-assay variability obtained in the laboratory with the internal quality controls was $3.9 \%$ at $45.7 \mu \mathrm{mol} / 1$ and $2.4 \%$ at $108 \mu \mathrm{mol} / 11$ the intra-assay variability was $3.3 \%$ at $44.5 \mu \mathrm{mol} / 1$ and $0.7 \%$ at $148 \mu \mathrm{mol} / 1$. The laboratory is participating in an external quality assessment scheme.

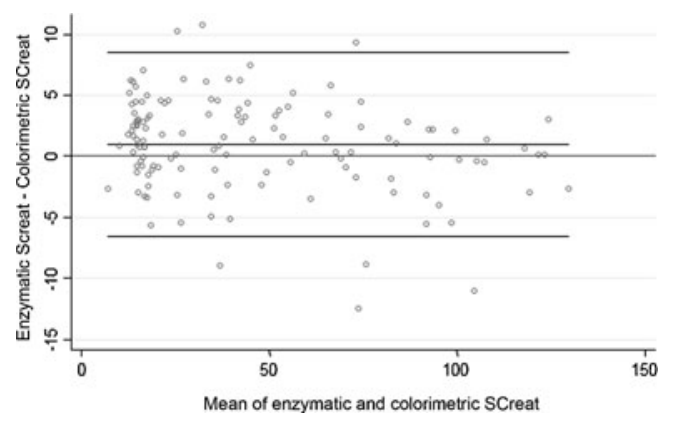

Fig. 1 Correlation between enzymatic and colorimetric serum creatinine (SCr)

\section{Measured GFR method}

Measured GFR (mGFR) was obtained by the clearance of inulin [Inutest, $25 \%$ (sinistrin); Fresenius Kabi, Bad Homburg, Germany). Inulin clearance (iGFR) is still considered the gold standard for GFR measurement, as inulin is freely filtrated through the glomerulus and is neither secreted nor reabsorbed by the tubule. In our study, inulin clearance was obtained by a standard technique used by a trained staff as follows. Two intravenous catheters were inserted, one in each arm. The administration of a loading dose of inulin (Inutest $25 \%$; Fresenius, Austria) $30 \mathrm{mg} / \mathrm{kg} \mathrm{BW}$ was followed by a continuous infusion for $3 \mathrm{~h}$, with the aim of obtaining a constant plasma concentration of between 200 and $500 \mathrm{mg} / \mathrm{l}$ of inulin. Water diuresis was induced by the oral administration of $20 \mathrm{ml} / \mathrm{kg}$ of water (maximum 1,200 ml) in the first hour, followed by $3 \mathrm{ml} / \mathrm{kg} \mathrm{BW}$ of water, in combination with an intravenous infusion of $0.9 \%$ sodium chloride (maximum $300 \mathrm{ml}$ ) every $30 \mathrm{~min}$. After a 90-min equilibration period, three timed-urine samples were collected every $30 \mathrm{~min}$, with a blood test performed in the middle of each urine collection. Inulin was measured using the automatic anthrone method of Wright and Gann [8] on an Autoanalyzer 3 system (high-resolution digital colorimeter; SEAL, Bran + Luebbe, Norderstedt, Germany; now SEAL, Burgess Hill, UK). The method was calibrated with five-points calibration (target values at $10,20,30,40$, and $50 \mathrm{mg} / 100 \mathrm{ml}$ ) with coefficient correlations of $0.9993 \pm 0.0005$. The procedure is automatized with software (AACE ver. 6.03; Bran + Luebbe) which automatically includes corrections for baseline, carryover, sensitivity drift, and dilution factor. For the serum, the intra-assay variability coefficients obtained in our laboratory with the internal quality controls were $2.44 \%$ at $10 \mathrm{mg} / 100 \mathrm{ml}, 1.47 \%$ at $30 \mathrm{mg} / 100 \mathrm{ml}$, and $0.94 \%$ at $40 \mathrm{mg} / 100 \mathrm{ml}$, while for urine, the intraassay variability coefficients were $1.71 \%$ at $10 \mathrm{mg} /$ $100 \mathrm{ml}, 1.22 \%$ at $30 \mathrm{mg} / 100 \mathrm{ml}$, and $1.07 \%$ at $50 \mathrm{mg} / 100 \mathrm{ml}$. The inter-assay variability coefficients 
obtained in the internal laboratory standards were $2.35 \%$ at $10 \mathrm{mg} / 100 \mathrm{ml}, 2.23 \%$ at $30 \mathrm{mg} / \mathrm{ml}$, and $0.87 \%$ at $50 \mathrm{mg} / 100 \mathrm{ml}$.

Inulin clearance glomerular filtration rate was calculated as the mean of the three clearance periods. When inulin clearance difference between 2 periods exceeded $20 \%$, that period was excluded, and iGFR was calculated as the mean of the 2 valid periods.

\section{Estimated GFR method}

The eGFR was calculated according to the MDRD formula, the CKD-EPI formula, and the Cockcroft-Gault formula and expressed in milliliters per minute per $1.73 \mathrm{~m}^{2}$ as follows:

- MDRD formula: eGFR $=175 \times \mathrm{SCr}^{-1.154} \times \mathrm{age}^{-0.203} \times$ [0.742 if female] $\times$ [1.212 if black], where $\mathrm{SCr}$ measured by an IDMS-calibrated assay is in milligrams per deciliter and age is in years.

- CKD-EPI formula: eGFR $=a \times(\mathrm{Scr} / b)^{c} \times(0.993)^{\mathrm{age}} \times$ $0.993^{\text {age }}$. The variable $a$ takes the following values on the basis of race and sex: black women, 166; black men, 163; white/other women, 144; white/other men, 141. The variable $b$ takes on the following values on the basis of sex: women, 0.7; men, 0.9. The variable $c$ takes on the following values on the basis of sex and SCr measurement: for women, if $\mathrm{SCr} \leq 0.7 \mathrm{mg} / \mathrm{dl}=-0.329$; if $\mathrm{SCr}>0.7 \mathrm{mg} / \mathrm{dl}=-1.209$. For men, if $\mathrm{SCr} \leq 0.9 \mathrm{mg} / \mathrm{dl}$ $=-0.411 ;$ if $\mathrm{SCr}>0.9 \mathrm{mg} / \mathrm{dl}=-1.209$.

- Cockcroft-Gault formula: eGFR $=(140-$ age $\times \mathrm{BW} \times$ $[\mathrm{K}=1.23$ if male $] \times[\mathrm{K}=1.05$ if female $] / \mathrm{SCr}$, where age is in years, $\mathrm{BW}$ in kilograms and $\mathrm{SCr}$ in micromole per liter. This formula was established using the old $\mathrm{Cr}$ measurement method (Jaffe technique). We used the compensated Jaffe technique to measure $\mathrm{Cr}$, adjusted to the new enzymatic method. In order to avoid any bias due to using the Cockcroft-Gault published constants (1.23 for males of any age, and 1.05 for females of any age), which can lead to an inaccurate estimation of GFR, we calculated new constants based on the iGFR for each patient and then calculated the average. The recalculated average constants were $1.10 \pm 0.34$ and $1.16 \pm 0.32$ for female and male children, respectively. We then compared both the Cockcroft-Gault formula using the published constants and the modified Cockcroft-Gault formula using the newly established constants to the iGFR.

\section{Statistical analysis}

Data analysis was performed using STATA ver. 12.0 (StataCorp, College Station, TX). Population characteristics (age, sex, weight and height in age independent $Z$ scores) were compared between CKD groups using Kruskal-Wallis equality-of-populations rank test or chi-squared test depending on the type of variable. Each formula was compared to iGFR in term of bias, accuracy, correlation, and precision. Bias (difference between eGFR and iGFR values) were assessed using limits of agreement on the Bland and Altman plot. For the MDRD, Cockcroft-Gault, and modified Cockcroft-Gault formulas, one of the assumptions when using such limits of agreements was not reached (homoscedasticity). Therefore, in order to evaluate if the eGFR and iGFR values differed and to be able to draw conclusions on bias, we used the Wilcoxon Signed-Rank test. Accuracy was defined as the percentage of eGFR values that were within 10 and $30 \%$ of the iGFR. Correlation was analyzed with the Spearman correlation coefficient. Precision was defined as the percentage of variance of the eGFR explained by the iGFR obtained through a multiple linear regression with age and sex as control covariates. A log transformation of the eGFR (for the MDRD, Cockcroft-Gault, and modified Cockcroft-Gault formulas) and iGFR was used to reach the assumption of application; therefore, Lin's coefficient was not used as is common. Significance was defined as $p \leq 0.05$, and $p$ values were corrected for multiple testing.

\section{Results}

The three groups differ in terms of Ht $(p<0.001)$ and BW $(p<0.001)$ age-independent $Z$ scores. The children in group 3 have a standardized $\mathrm{Ht}$ that is smaller than that of children in group $2(p<0.001)$ and group $1(p<0.001)$. The children in group 3 have a standardized BW that is lower than that of children in group $2(p<0.001)$ and group $1(p<0.001)$; the standardized BW for children in group 2 is also lower than that of group 1 children $(p=0.027)$. There are more females in group 3 than in group 1 (58 vs. $42 \%, p=0.02)$ and group $2(58$ vs. $43 \%, p=0.023)$. Children in group 1 are younger than those in group $2(p=0.019)$ and 3 $(p=0.011)$. Medians with the upper and lower quartiles [25\%; $75 \%$ ] of iGFRs and eGFRs using all of the formulas studied here are summarized in Table 3.

Comparison of eGFR using the MDRD formula and iGFR

As shown in Table 4, there is a significant bias between the eGFR and iGFR for each CKD group $(p<0.001)$. The medians of bias between eGFR and iGFR are significantly different from zero $(p<0.001)$ and also decrease significantly with worsening CKD $\left(74,57\right.$ and $23 \mathrm{ml} / \mathrm{min} / 1.73 \mathrm{~m}^{2}$ for groups 1, 2 and 3, respectively; $p<0.01$ between groups). Furthermore, the accuracy is poor: with an accuracy of 10 and $30 \%$, only 11 and $25 \%$ of the eGFRs are accurate, respectively (values are similar for the different CKD groups). 
Table 3 Distribution of the medians with upper and lower quartiles of iGFRs and eGFRs using the studied formulas, in all patients and in each group of chronic kidney disease (CKD)

\begin{tabular}{lcccc}
\hline $\begin{array}{l}\text { Median iGFR and eGFRs calculated using the studied formulas } \\
\left(\mathrm{ml} / \mathrm{min} / 1.73 \mathrm{~m}^{2}\right)\end{array}$ & All patients & $\begin{array}{l}\text { Group 1: CKD } \\
\text { stage I }\end{array}$ & $\begin{array}{l}\text { Group 2: CKD } \\
\text { stage II }\end{array}$ & $\begin{array}{l}\text { Group 3: CKD stage III, } \\
\text { IV and V }\end{array}$ \\
\hline Median iGFR & $92[78 ; 103]$ & $102[95 ; 112]$ & $81[73 ; 86]$ & $42[31 ; 50]$ \\
Median eGFRs using MDRD formula & $148[106-200]$ & $175[133 ; 248]$ & $133[105 ; 174]$ & $59[44 ; 90]$ \\
Median eGFRs using CKD-EPI formula & $141[118 ; 155]$ & $149[135 ; 167]$ & $136[118 ; 148]$ & $64[48 ; 99]$ \\
Median eGFRs using Cockcroft-Gault formula & $93[75 ; 115]$ & $103[86 ; 124]$ & $90[75 ; 111]$ & $43[32 ; 54]$ \\
Median eGFRs using modified Cockcroft-Gault formula & $91[75 ; 114]$ & $100[84 ; 123]$ & $87[73 ; 109]$ & $42[33 ; 52]$ \\
\hline
\end{tabular}

CKD-EPI, chronic kidney disease epidemiology collaboration; eGFR, estimated glomerular filtration rate; iGFR inulin glomerular filtration rate; MDRD, modification of diet in renal disease

Values presented in square parenthesis are the upper and lower quartiles [25\%; $75 \%$ ]

In terms of the correlation between eGFR and iGFR, we were expecting a correlation close to 1 for an instrument measuring the same outcome. In contrast, we found that there is a significant but poor correlation between the eGFR and the iGFR for all patients(Spearman correlation coefficient of $0.55, p<0.001)$. When the correlation in each group was analyzed separately, we did find a significant correlation between the eGFR and the iGFR in groups 1 and 3, with a Spearman correlation coefficient of 0.32 and 0.65 , respectively $(p<0.001)$, but again this correlation is poor. We found no significant correlation between the eGFR and the iGFR in group 2 (Spearman correlation of $0.11, p=0.13$ ). The correlation between the eGFR and iGFR values is presented in Fig. 2a. If both measures were to be close to each other, the bias will be not significant and the correlation will be close to 1 , which is represented by the solid line. However, bias can be seen to be significantly greater than 0 , which is observed as a significant overestimation of the iGFR when the MDRD formula is applied. The Bland-Altman plot of the relationship between the percentage errors of the MDRD formula with the measured GFRs is shown in Fig. $3 \mathrm{a}$.

It should be noted that the MDRD formula was first derived from the analysis of patients with an mGFR of $<60 \mathrm{ml} / \mathrm{min} / 1.73 \mathrm{~m}^{2}$ (i.e., CDK stages III, IV, and V), for whom we obtain the best correlation, a better precision [better standard deviation (SD) of bias], and the lowest median difference, which is significantly lower than that for CDK stage I $(p<0.001)$ and CDK stage II $(p<0.001)$.

In order to test the precision of the MDRD formula, we modelized eGFR. The variability of eGFR was found to increase with higher iGRF values, and, therefore, we logtransformed eGFR and iGFR. Only $48 \%$ of the variation of the log eGFR is explained by the log iGFR. After controlling for age, the explained variance of log eGFR increases up to $82 \%$, showing an inverse correlation between age and log eGFR. Controlling for gender increases the explained variance by $4 \%$, with boys having a mean of log eGFR higher than girls.
Comparison of eGFR using the CKD-EPI formula and iGFR

The analysis of the eGFR obtained using the CKD-EPI formula is summarized in Table 4 . There is a significant bias between eGFR and iGFR $(p<0.001)$ in the whole population and also in each CKD group. Medians of bias between eGFR and iGFR are significantly different from zero and also different between CKD groups (46, 57 and $27 \mathrm{ml} / \mathrm{min} / 1.73 \mathrm{~m}^{2}$ for groups 1,2 , and 3, respectively, with $p<0.01$ between groups). The accuracy is also poor: with an accuracy of 10 and $30 \%$, only 6 and $20 \%$, respectively, of the eGFRs are accurate (the accuracy in group 1 is greater than in the other groups).

The Spearman correlation coefficient $(r)$ between eGFR and iGFR is significant $(p<0.001)$ but poor $(r=0.55)$ for the whole cohort and also for group $1(p<0.001, r=0.32)$. This correlation is significant and higher for group $3(p<0.001$, $r=0.69$ ), but there is low accuracy and also low precision $(\mathrm{SD}$ of bias $=28)$. In group 2, the Spearman correlation between eGFR and iGFR is not significant, with a poor coefficient ( $p=0.104, r=0.12)$.

Figure $2 \mathrm{~b}$ presents the correlation between the values of eGFR and iGFR. If both measures were to be close, the bias will be not significant and the correlation will be close to 1 , which is represented by the solid line. Bias is significantly greater than 0 , which can be observed as a significant overestimation of the iGFR when the CKD-EPI formula is applied. The Bland-Altman plot of the percentage error of eGFRs with respect to the iGFRs is shown in Fig. 3b. The Spearman correlation coefficient between the relative bias and iGFR is significant in the whole sample and in groups 1 and 2 (Table 4).

In order to test the precision of the CKD-EPI formula, we modelized eGFR with a linear regression and found that $50 \%$ of the variation of the eGFR is explained by the iGFR. Controlling for age increases the explained variance of eGFR up to $74 \%$, showing an inverse correlation between age and eGFR. Controlling for gender increases the 


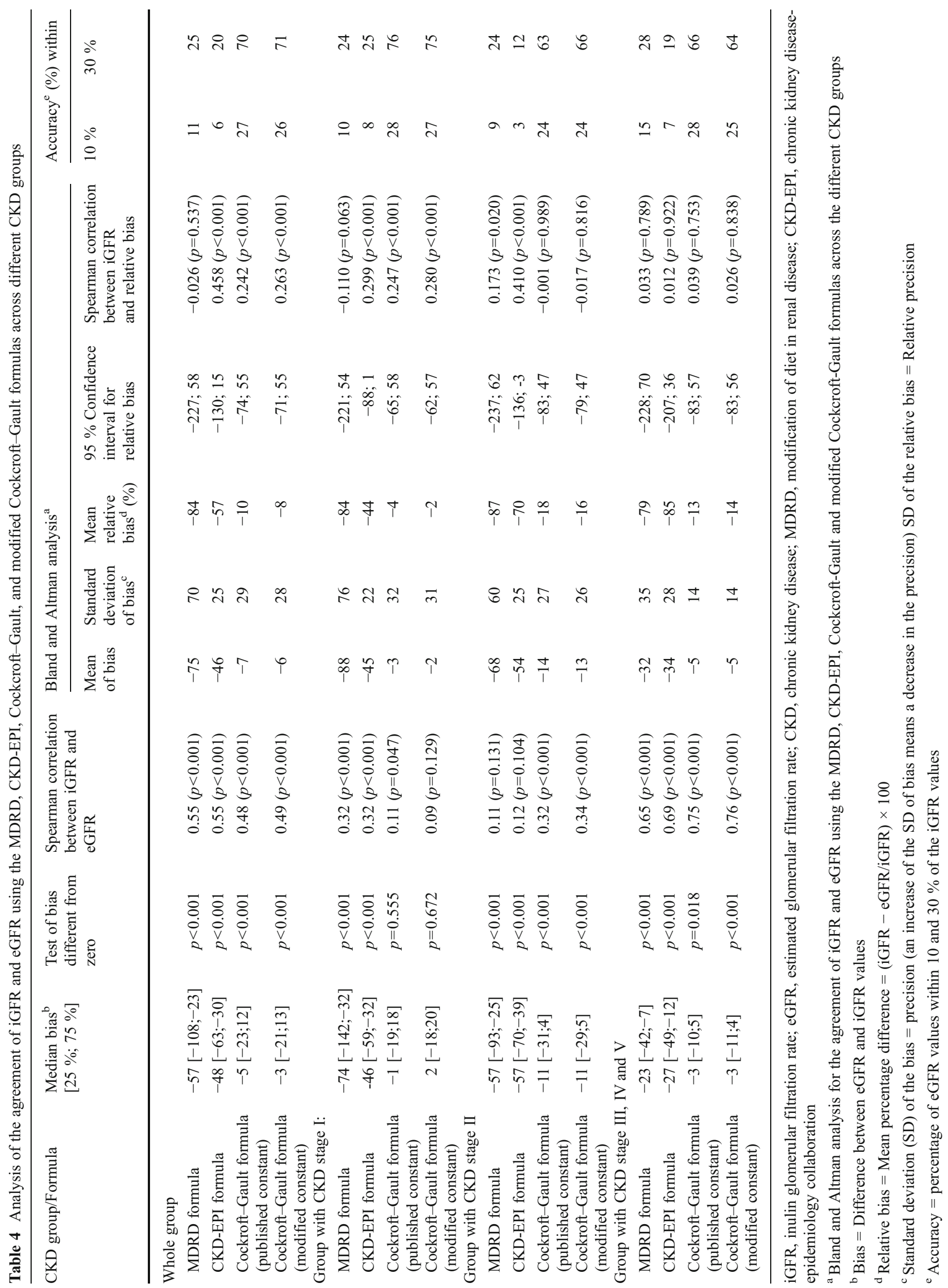


Fig. 2 Correlation between inulin GFR (iGFR) and eGFR calculated using the modification of diet in renal disease (MDRD), the chronic kidney disease-epidemiology collaboration (CKD-EPI), the Cockcroft-Gault, and the modified Cockcroft-Gault formulas, respectively. Solid line represents the line of identity between iGRF and eGFR, dashed, dotted, and dot-dashed lines indicate $\pm 10,30$ and $50 \%$ deviations, respectively. eGFR, estimated glomerular filtration rate; iGFR, inulin clearance glomerular filtration rate
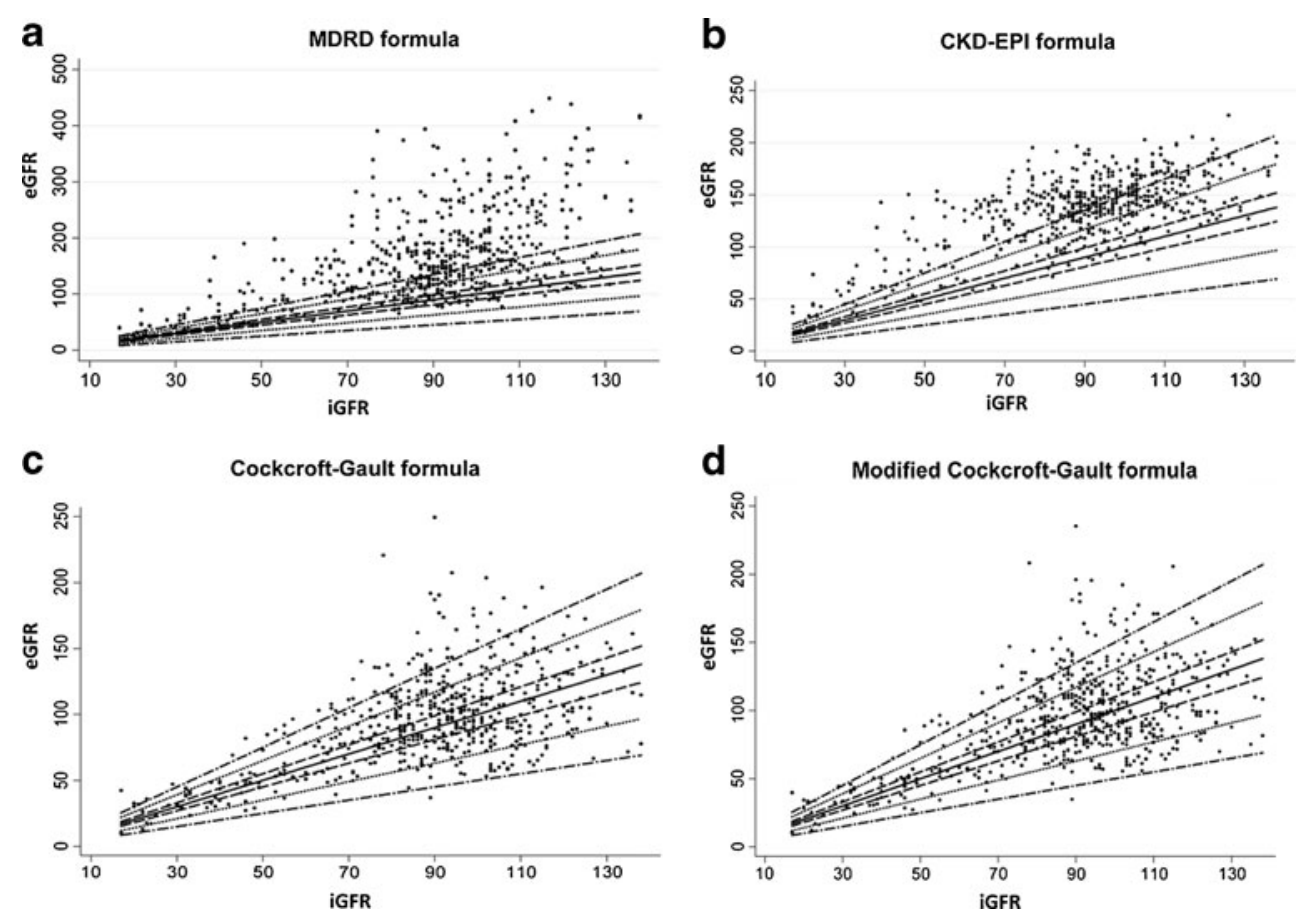

explained variance by $4 \%$, with boys having a mean of eGFR higher than girls.

Comparison of eGFR using the Cockcroft-Gault formula and iGFR

For the Cockcroft-Gault formula, the median of bias between iGFR and eGFR (Table 4) is significantly different from zero for the whole cohort, and also the Spearman correlation is poor $(r=0.48)$. In addition, with an accuracy of $10 \%$ and $30 \%$, only $27 \%$ and $70 \%$ of the eGFRs are accurate respectively. These accuracies are below the KDOQI targets.

When analyzing the subgroups in details, we found that the bias is significantly different from zero for groups 2 and 3 . For children in group 1, the bias is
Fig. 3 Relationship between the percentage errors of different eGFR formulas and iGFR using the Bland-Altman plot. Percentage error $=(\mathrm{iGFR}$ $-\mathrm{eGFR} / \mathrm{iGFR}) \times 100$.

Spearman correlation coefficient $(r)$ between the percentage error and iGFR is presented in Table 4. eGFR, estimated glomerular filtration rate; iGFR, inulin clearance glomerular filtration rate
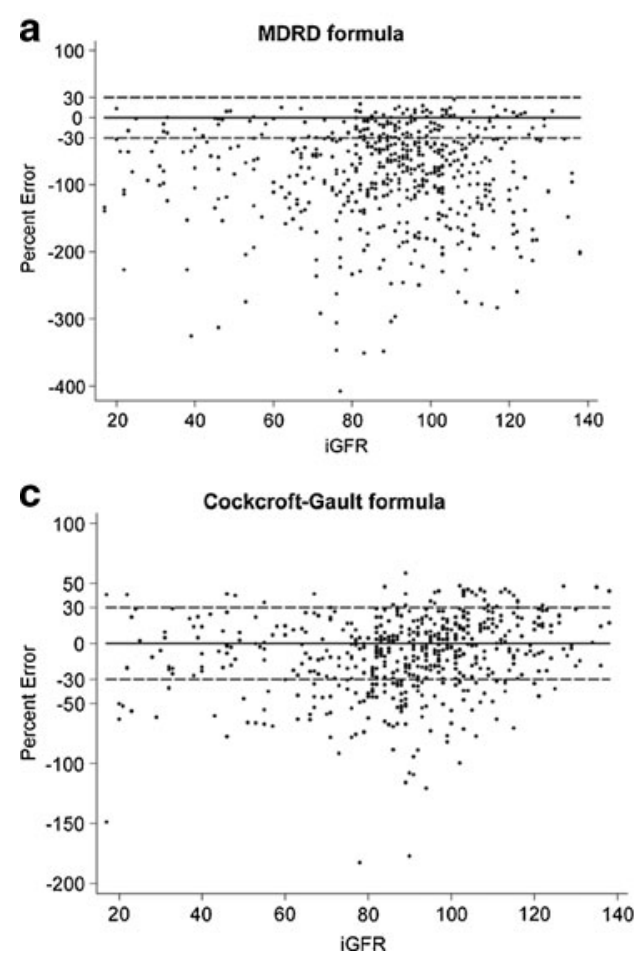
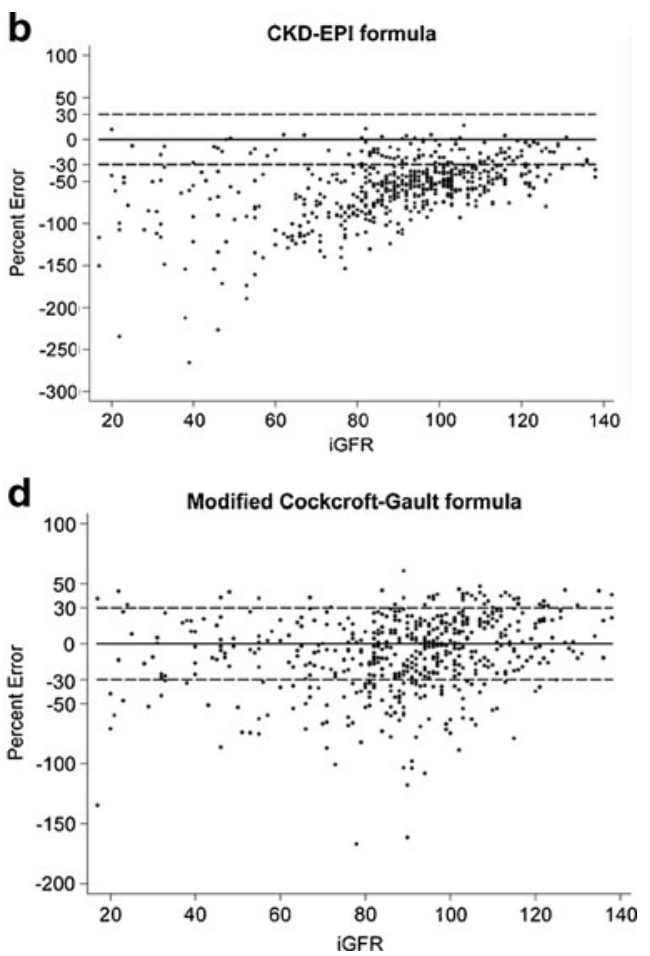
not significantly different from zero, but there is a very poor correlation between iGFR and eGFR $(r=0.114)$ and the correlation between the percentage error and iGFR is significant $(r=0.25, p<0.001)$.

The correlation between eGFR and iGFR values is presented in Fig. 2c, where we observe an overestimation of the iGFR when applying the Cockcroft-Gault formula. The Bland-Altman plot of the percentage error of eGFRs with respect to the iGFRs is shown in Fig. 3c.

In order to test the precision of Cockcroft-Gault formula, we modelized eGFR. The variability of eGFR increases with higher iGFR values, we therefore log-transformed eGFR and iGFR. Only $53 \%$ of the variation of the log eGFR is explained by the log iGFR. After controlling for age, the explained variance of $\log$ eGFR increases up to $70 \%$, showing a positive correlation between age and log eGFR. Controlling for gender adds $2 \%$ of explained variance with boys having a mean of log eGFR higher than girls.

Comparison between eGFR using the modified Cockcroft-Gault formula and iGFR

Comparisons between iGFR and the Cockcroft-Gault formula, modified or not, are very similar. Table 4 shows that the median of bias between iGFR and eGFR is significantly different from zero for the whole cohort, with a Spearman correlation coefficient of 0.49. The accuracy of the modified Cockcroft-Gault formula is poor: with an accuracy of 10 and $30 \%$, only 26 and $71 \%$, respectively, of the eGFRs are accurate. When analyzing the subgroups, we found that the accuracy decreases with CKD progression and that the median of bias is significantly different from zero for groups 2 and 3. For children in group 1, although the median of bias is not significantly different from zero, the correlation between iGFR and eGFR is close to zero $(r=0.09)$, and there is a significant correlation between the percentage error and iGFR $(r=0.28, p<0.001)$.

Figure $2 \mathrm{~d}$ presents the relation between the values of eGFR and iGFR where we observe an overestimation of the iGFR when applying the modified Cockcroft-Gault formula. The Bland-Altman plot of the percentage error of eGFRs with respect to the iGFR is shown in Fig. 3d. The Spearman correlation coefficient between the relative bias and iGFR is significant in the whole sample and in group 1 (Table 4).

\section{Discussion}

Serum creatinine-based formulas are the most widely used formulas for estimating the GFR [9]. Formulas for eGFR in adults were developed in the 1970s. The first of these was the Cockcroft-Gault formula [3], which was developed exclusively in a white male population. This formula was approved in 1998 by the U.S. Food and Drug Administration to guide drug dosing for patients with decreased kidney function [10]; however, it was not adequately adapted for sex and race. A new equation, the MDRD formula, was published in 1999 [4] and subsequently simplified in 2000 [11]. The most commonly used MDRD formula is the simplified "4-variable MDRD," which estimates GFR using four variables: $\mathrm{SCr}$, age, race, and sex. The simplified 4variable MDRD prediction formula performs as well as the more complicated original six-variable MDRD formula, which also includes blood urea nitrogen and albumin levels. This formula was developed in a group of adult patients with a mean age of 51 years and a mean GFR of $40 \mathrm{ml} / \mathrm{min} /$ $1.73 \mathrm{~m}^{2}$ and was subsequently evaluated and validated in African-Americans with CKD [12], in patients with scleroderma [13], in potential kidney donors with a GFR of $<80 \mathrm{ml} / \mathrm{min} / 1.73 \mathrm{~m}^{2}$ [14], in patients with CKD and a normal SCr level [15], in 46 normal and 46 type 1 diabetes individuals without nephropathy [16], and in adults without renal disease [17]. The MDRD formula has proved to be accurate for patients with stage $3 \mathrm{CKD}$ or higher and less accurate for higher GFR levels.

The CKD-EPI formula, which was developed to overcome the limitations of the MDRD formula, was developed in a group of adults with a mean age of 47 years and a mean GFR of $67 \mathrm{ml} / \mathrm{min} / 1.73 \mathrm{~m}^{2}$. It has been externally validated in a group of patients with mild CKD and an average measured GFR of $<90 \mathrm{ml} / \mathrm{min} / 1.73 \mathrm{~m}^{2}$, and also in potential kidney donors. The CKD-EPI formula includes a two-level variable of race (black vs. white/other). A new version of this equation that includes a four-level race/ethnicity variable (Black, Asian, Native American, Hispanics, Whites, Others) has been developed, but has failed to show sufficient accuracy in daily clinical practice [18].

The National Kidney Foundation Kidney Disease Outcomes Quality Initiative (NKF-KDOQI) guidelines recommend using the MDRD formula for eGFR in adult patients at risk of kidney diseases. Expecting that it would not be ideal for estimating GFR in children, the National Kidney Foundation did not recommend using the MDRD formula in the pediatric population [19, 20]. Various formulas have been developed for infants, but all are characterized by several limitations. The most widely used formula in the pediatric population is the Schwartz formula, which was developed in the mid-1970s and revised in 2009 [21]. Unlike formulas for estimating the GFR in adults, the Schwartz formula was developed from a selected group of children with an mGFR of between 15 and $75 \mathrm{ml} / \mathrm{min}$ per $1.73 \mathrm{~m}^{2}$.

Here, we have evaluated the external validity of the MDRD, the CKD-EPI, and also the Cockcroft-Gault 
formulas based on plasma $\mathrm{Cr}$ in a pediatric population. Our results show that all of these formulas have several limitations in that age group. The MDRD and the CKD-EPI formulas significantly overestimate the mGFR and present variable accuracies depending on the specific CKD group. The MDRD formula shows a similar trend in children and adults, i.e., the eGFR is more accurate for an mGFR of $<60 \mathrm{ml} / \mathrm{min} / 1.73 \mathrm{~m}^{2}$. However, the accuracy of this formula in the pediatric population is very low compared to its accuracy in the adult population. In children, the CKD-EPI formula is more accurate for an $\mathrm{mGFR}$ of $>60 \mathrm{ml} / \mathrm{min} /$ $1.73 \mathrm{~m}^{2}$, which is also found in adults, but unfortunately it gives a poor estimation of GFR (low accuracy) in all children with CKD. We found that the Cockcroft-Gault formula performs slightly better than the MDRD and the CKD-EPI formulas for estimating GFR in children. However, this formula is still inaccurate when both the published constants or our recalculated constants are used. This has also been shown by Filler et al. [7]. In contrast, Pierrat et al. [6] demonstrated an acceptable estimation of GFR with the Cockcroft-Gault formula, but only in children above 12 years of age. Our results also show that when we modelized eGFR, age plays an important role in the eGFR imprecision/variability when applying the MDRD, CKDEPI and Cockcroft-Gault formulas.

Two forms of the MDRD equation are available, depending on whether or not $\mathrm{Cr}$ is measured by an IDMS-calibrated assay. The CKD-EPI equation is designed to be used with IDMScalibrated $\mathrm{SCr}$ values only. Also, in order to solve the major problem of the calibration of $\mathrm{SCr}$ measurement, our plasma $\mathrm{Cr}$ assays were performed in one laboratory with one method (compensated Jaffe technique) standardized to the enzymatic method, which is also standardized to be traceable to IDMS.

Our study has a number of limitations. First, we did not evaluate any formula that included cystatin C. Equations incorporating cystatin $\mathrm{C}$ have been developed recently [22, 23] and show various levels of accuracy when compared to mGFR. Cystatin $\mathrm{C}$ was not routinely measured in our patients, and therefore no comparison of formulas using Cystatin C to iGFR could be performed. Secondly, some patients were included twice in the study. However, these patients were included several years apart, with a minimum time frame of 2 years between the two assessments. Given major demographic differences between the two assessments (age, height, and renal disease progressions), this limitation should not weaken our findings.

In conclusion, the performance of the MDRD, CKD-EPI, and Cockcroft-Gault formulas against iGFR is unreliable across all CKD stages, in children of all ages. The use of these formulas in this population group is associated with accuracies far below the KDOQI targets of $\geq 90 \%$ needed for validation. These formulas cannot be applied in children, therefore confirming the NKF-KDOQI recommendations.

\section{References}

1. Vassalotti JA, Stevens LA, Levey AS (2007) Testing for chronic kidney disease: a position statement from the National Kidney Foundation. Am J Kidney Dis 50:169-180

2. Madero M, Sarnak MJ (2011) Creatinine-based formulae for estimating glomerular filtration rate: is it time to change to chronic kidney disease epidemiology collaboration equation? Curr Opin Nephrol Hypertens 20:622-630

3. Cockroft DW, Gault MH (1976) Prediction of creatinine clearance from serum creatinine. Nephron 16:31-41

4. Levey AS, Bosch JP, Lewis JB, Greene T, Rogers N, Roth D (1999) A more accurate method to estimate glomerular filtration rate from serum creatinine: a new prediction equation. Modification of Diet in Renal Disease Study Group. Ann Intern Med 130:461-470

5. Levey AS, Stevens LA, Schmid CH, Zhang YL, Castro AF 3rd, Feldman HI, Kusek JW, Eggers P, Van Lente F, Greene T, Coresh J, CKD-EPI (Chronic Kidney Disease Epidemiology Collaboration) (2009) A new equation to estimate glomerular filtration rate. Ann Intern Med 150:604-612

6. Pierrat A, Gravier E, Saunders C, Caira MV, Aït-Djafer Z, Legras B, Mallié JP (2003) Predicting GFR in children and adults: a comparison of the Cockcroft-Gault, Schwartz, and Modification of diet in renal disease formulas. Kidney Int 64:1425-1436

7. Filler G, Foster J, Acker A, Lepage N, Akbari A, Ehrich JH (2005) The Cockcroft-Gault formula should not be used in children. Kidney Int 67:2321-2324

8. Wright HK, Gann DS (1966) An automatic anthrone method for determination of inulin in plasma and urine. J Lab Clin Med 67:689-693

9. Hogg RJ, Furth S, Lemley KV, Portman R, Schwartz GJ, Coresh J, Balk E, Lau J, Levin A, Kausz AT, Eknoyan G, Levey AS (2003) National Kidney Foundation's Kidney Disease Outcomes Quality Initiative clinical practice guidelines for chronic kidney disease in children and adolescents: evaluation, classification, and stratification. Pediatrics 111:1416-1421

10. Food and Drug Administration, U.S. Department of Health and Human Services (2010) Guidance for industry: pharmacokinetics in patients with impaired renal function: study design, data analysis, and impact on dosing and labeling. Available at: www.fda.gov/ downloads/Drugs/GuidanceComplianceRegulatoryInformation/ Guidances/UCM72127.pdf. Accessed 5 Feb 2010

11. Levey AS, Greene T, Kusek JW, Beck GJ (2000) A simplified equation to predict glomerular filtration rate from serum creatinine. J Am Soc Nephrol 11:A0828

12. Lewis J, Agodoa L, Cheek D, Greene T, Middleton J, O'Connor D, Ojo A, Phillips R, Sika M, Wright J Jr, African-American Study of Hypertension and Kidney Disease (2001) Comparison of crosssectional renal function measurements in African Americans with hypertensive nephrosclerosis and of primary formulas to estimate glomerular filtration rate. Am J Kidney Dis 38:744-753

13. Kingdon EJ, Knight CJ, Dustan K, Irwin AG, Thomas M, Powis SH, Burns A, Hilson AJ, Black CM (2003) Calculated glomerular filtration rate is a useful screening tool to identify scleroderma patients with renal impairment. Rheumatology 42:26-33

14. Bertolatus JA, Goddard L (2001) Evaluation of renal function in potential living kidney donors. Transplantation 71:256-260

15. Bostom AG, Kronenberg F, Ritz E (2002) Predictive performance of renal function equations for patients with chronic kidney disease and normal serum creatinine levels. J Am Soc Nephrol 13:2140 2144

16. Vervoort G, Willems HL, Wetzels JF (2002) Assessment of glomerular filtration rate in healthy subjects and normoalbuminuric diabetic patients: validity of a new (MDRD) prediction equation. Nephrol Dial Transplant 17:1909-1913 
17. Lin J, Knight EL, Hogan ML, Singh AK (2003) A comparison of prediction equations for estimating glomerular filtration rate in adults without kidney disease. J Am Soc Nephrol 14:25732580

18. Stevens LA, Claybon MA, Schmid CH, Chen J, Horio M, Imai E, Nelson RG, Van Deventer M, Wang HY, Zuo L, Zhang YL, Levey AS (2011) Evaluation of the Chronic Kidney Disease Epidemiology Collaboration equation for estimating the glomerular filtration rate in multiple ethnicities. Kidney Int 79:555-562

19. Levey AS, Coresh J (2002) K/DOQI clinical practice guidelines on chronic kidney disease. Guideline 4. Estimation of GFR. Am J Kidney Dis 3976-92

20. National Kidney Foundation (2002) K/DOQI clinical practice guidelines for chronic kidney disease: evaluation, classification, and stratification. Part 5. Evaluation of laboratory measurements for clinical assessment of kidney disease. Guideline 4. Estimation of GFR. Available at: http://www.kidney.org/professionals/doqi/ kdoqi/guidelines_ckd/p5_lab_g4.htm

21. Schwartz GJ, Muñoz A, Schneider MF, Mak RH, Kaskel F, Warady BA, Furth SL (2009) New equations to estimate GFR in children with CKD. J Am Soc Nephrol 20:629-637

22. Grubb A, Nyman U, Björk J, Lindström V, Rippe B, Sterner G, Christensson A (2005) Simple cystatin C-based prediction equations for glomerular filtration rate compared with the modification of diet in renal disease prediction equation for adults and the Schwartz and the Counahan-Barratt prediction equations for children. Clin Chem 51:1420-1431

23. Sharma AP, Yasin A, Garg AX, Filler G (2011) Diagnostic accuracy of cystatin C-Based eGFR equations at different GFR levels in children. Clin J Am Soc Nephrol 6:1599-1608 